\title{
Hall Effect Sensor for Animal Geomagnetic Navigation
}

\section{Sachs $\mathrm{F}^{*}$}

Department of Physiology and Biophysics, University at Buffalo, USA

*Corresponding author: Frederick Sachs, Department of Physiology and Biophysics, University at Buffalo, NY 14214, USA, Tel: 716829 5161; Email: Sachs@buffalo.edu

\section{Opinion \\ Volume 4 Issue 2}

Received Date: July 21, 2021

Published Date: July 30, 2021

DOI: $10.23880 /$ aabsc- 16000165

\section{Introduction}

The magnetic compass has been a valuable human navigation tool for hundreds of years. Yet animals are known to align themselves with the earth's magnetic field without using ferrimagnets [1]. How do they do that? A number of models have been proposed but most are magnetic field sensors but lack the vectorial properties that allow telling north from south. Since animals are diamagnetic it is not obvious how they can tell north from south. Any sensory system to do this must be asymmetric, and there is one well known and commonly used diamagnetic sensor, the Hall Effect, whereby a charge flow in one direction with an orthogonal B field will induce a further orthogonal potential.

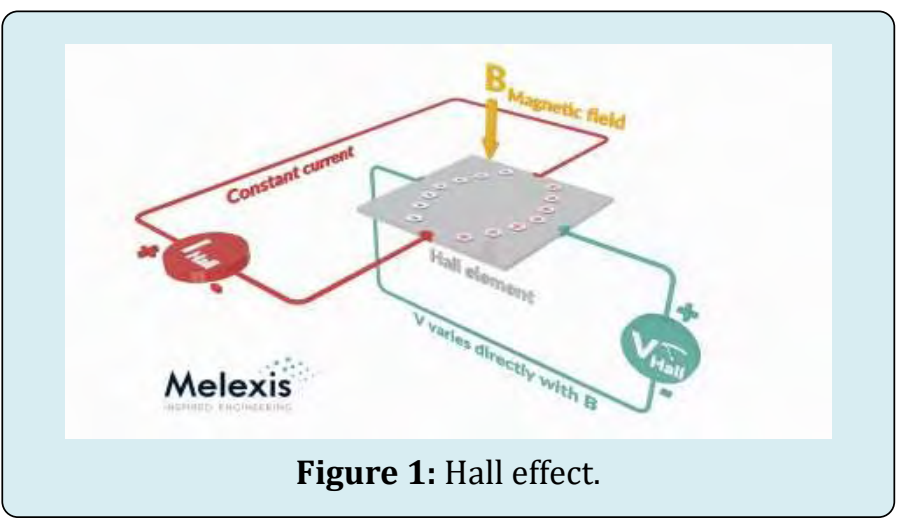

This Hall effect is commonly used in commercial magnetic field sensors with silicon microfabrication [2]. The vectorial nature of the interaction allows the sensor to respond to the direction of the $B$ field. Did animals ignore the availability of this potential compass?

For a simple rectangular sensor as shown above, the Hall voltage, $\mathrm{VH}$, is given by:

$$
V h=I B / q n d \quad \text { Eq1 }
$$

Where I is the current flowing through the sensor; B is the magnetic field strength, $q$ is the carrier charge; $n$ is the number of charge carriers per unit volume; $d$ is the width of the sensor. As a simple approximation for biology, consider a nerve fiber $10 \mu \mathrm{m}$ in diameter carrying $1 \mu \mathrm{A}$ of current in an earth's magnetic field of $50 \mu \mathrm{T}, \mathrm{Vh} \sim 3^{*} 10^{-14} \mathrm{~V}$ (https:// fxsolver.com). This amplitude is too small to be detected in a biological system. What can we change?

The sensor in an animal is not likely to be a local organelle, but a distributed component with defined axes. There is not much we can change in the nerve calculation to significantly increase the amplitude. However, if we consider a blood vessel that contains many movable charges, the Hall potential is given by (https://pressbooks.uiowa.edu/ clonedbook/chapter/the-hall-effect/),

$$
V h=B d v
$$

Where the vessel diameter is $d$ and the flow velocity is v. In the median earth's field, a medium sized vessel with $d=4 \mathrm{~mm}$ and $v=0.2 \mathrm{~m} / \mathrm{s}$, provides $40 \mathrm{nV}$. Marine fish are capable of detecting gradients as small as $5 \mathrm{nV} / \mathrm{cm}$ ! [3]. Presumably if the vessel were efficiently linked to sensory nerves, the signal could be transduced, with phase detection via the heartbeat. A further reduction in noise sensitivity could be handled by combining the signals from orthogonal vessels in a bridge format. This should allow the animal to detect the direction of the field as well as its magnitude. Now it is time for experiments!

\section{References}

1. Zhao Z, Hu T, Cui W, Huangfu J, Li C (2014) Long-distance geomagnetic navigation: Imitations of animal migration based on a new assumption. IEEE Transactions on Geoscience and Remote Sensing 52(10): 6715-6723. 
2. Lozanova SV, Roumenin CS (2009) Parallel-field silicon Hall effect microsensors with minimal design complexity. IEEE Sensors Journal 9(7): 761-766.
3. Kramer B (1996) Electroreception and communication in fishes, Gustav Fischer. 